\title{
Reflections on the Future Electric Power Grid Monitoring System
}

\author{
Michael Gouzman and Serge Luryi \\ Department of Electrical and Computer Engineering, State University of New York at Stony Brook, \\ Stony Brook, NY 11794-2350 USA \\ Serge.Luryi@stonybrook.edu
}

Received 30 June 2014

Accepted 19 July 2014

\begin{abstract}
A shortcoming of the contemporary power grid monitoring is that the system does not know its own state. Instead of taking automatic note of energy-flow disruptions, one deals with haphazard telephone reports of "no light in our house". We propose a novel monitoring system that requires no restructuring of the power distribution network and can be applied both to the existing grids and the future "smart grids". The proposed system is based on a network of inexpensive sensors, installed on every connecting line and communicating measured data to a central processing unit. Our approach is topological in nature, based on the connectivity aspects of the power grid embodied in Kirchhoff's current law that must be valid at every node of the network. We argue that the state of the network can be adequately characterized by specifying the RMS currents and the direction of energy flow in all connecting lines. It is essential that in this description one does not have to know the magnitude of the energy flow, only its direction. This eliminates the need to measure voltage, which would be prohibitively costly on the massive scale. In contrast, the relative phase between the current and voltage can be measured easily. Another essential point is that the instantaneous RMS currents are impractical to record and communicate, hence local averaging is required. Since Kirchhoff's law should remain valid upon averaging, the latter must be carried out at each sensor synchronously over the entire network with global synchronization provided by the GPS.
\end{abstract}

Keywords: Sensors; grid topology; Kirchhoff's law.

\section{Introduction: Background and Desiderata}

Contemporary power grid distribution systems represent a legacy of more than 100 years of development. One of their most significant shortcomings is that the system does not know its own state. This is painfully felt during periods of massive failure caused by the fury of the elements or by a technical failure of a major component. Making our power distribution networks robust and rapidly recoverable after natural disasters is perhaps the most urgent task in the on-going improvement of the national infrastructure. The importance of this task is generally understood. Its key elements include implementing modern monitor and control systems for power networks. These two systems must work coherently (with due regard for each other) - with the monitor system setting the appropriate environment for the control system. 
What is there to monitor? This is a fair question. Each power distribution network can be described by a Graph represented by nodes of specified nature (such as generators, transformers, loads, switches, and storage units), their specified geographical position on a 2D map, and the topology of connecting lines. The State of the network is further characterized by the energy flow over the Graph.

Disruptions of the energy flow are associated with the breakdown of electrical connectivity of the power network and the required information that fully characterizes the network is therefore topological in nature. This is not, however, the shape in which the information about network failures reaches the control room of the contemporary utility. ${ }^{1}$ Instead of the desired automatic description of energy-flow disruptions, one deals with haphazard telephone reports of "no light in our house". The price we pay is in delayed and low-efficiency repair activity.

The electrical grid monitor system (EGMS) proposed in this work is based on the modern sensor, communication and computational technologies. The EGMS will not tamper with the power networks themselves and it can be applied both to the existing grids and the future smart grids. The sensor to be used must be cheap and safe. The latter requirement precludes direct electrical contact with "hot" wires, so that the smart measurements must be based on sensing the magnetic field.

Let us briefly discuss alternative approaches to automatic monitoring state of the power grid. The simplest imaginable system ${ }^{2}$ could be based on "on-off" reports of local access to power, an automated sensor-based version of the customer telephone call. ${ }^{3}$ Even when such reports are fully collected and accurate, they provide no information needed for a topological analysis of the possibly impaired connectivity of the grid.

On the other end of the scale, there exist sophisticated systems based on phasor measurement units (PMU). ${ }^{4}$ The PMU are sometimes called the synchrophasors, as they measure the instantaneous values of both the voltage and the current synchronously over the network. A PMU network can be compared to a multi-channel digital oscilloscope with synchronized channels in different locations. This is a very expensive proposition and todate PMU installation has been limited to critical substations of a power grid. ${ }^{5,6}$

Our vision of the EGMS aims at collecting the minimum data necessary to reconstruct the topological organization of the network. This makes our system inexpensive both in hardware and installation. The two innovations that lead to this cost reduction are $(i)$ abandoning instantaneous flow of data in favor of the average and (ii) eliminating all galvanic measurements, i.e. direct contact of sensors with the "hot" wire. Therefore, the EGMS can be contemplated to assume an utmost penetration of the power grid, down to single household units. We expect that individual sensors will cost less than $\$ 50$ and can be installed by the relatively unsophisticated electric utility personnel. We believe that utility companies will find the EGMS useful and practical.

As an added bonus, the EGMS provides a technical capability for apprehension of unwelcome leaks of the power from tapping of the grid by an unrecognized party. 


\section{Power Grid Topology}

Topology of a power grid is represented by a graph that shows connectivity of the electrical network. In operation, the state of the network can be characterized by the energy flow over the graph.

\subsection{Graph}

Power distribution network comprises Nodes connected by Connecting Lines. Each connecting line has at least two conducting wires. For a single-phase connecting line one of its two wires is the hot wire, and the other is ground wire. These phase wires must be appropriately matched with the respective Node.

Nodes of the network can be of different types, e.g. power sources, loads, power converters (transformers), and switches. The type of node is characterized by the direction of energy flow. A power source node is characterized by the outward direction of the energy flow in the associated connecting line. The load node is characterized by the inward direction of the energy flow in the associated connecting line. The power converter node typically has at least one inward and one outward connecting line. There can be also bidirectional nodes, such as rechargeable batteries.

The multiplicity of nodes and connecting lines form a Graph that is associated with a given power network.

\subsection{State of the network}

The state of the network is fully characterized by the energy flow over the Graph. The energy flow is impractical to monitor widely as it requires galvanic connections to highvoltage wires and therefore expensive sensors and sophisticated installation. We shall argue that the state of the network can be adequately characterized by specifying the RMS currents and the direction of energy flow in all connecting lines.

The energy flow depends on time which is reflected in the variation of instantaneous RMS currents. These variations are impractical to record, as they fluctuate on a short time scale. The reported RMS values must be pre-averaged over a specified time interval. It is essential that the averaging is carried out synchronously over the entire Graph.

The averaging periods must be long compared to the characteristic time of the fluctuations in the energy flows associated with random load variations. The energy flow in a particular high-level connecting line is analogous to the coordinate of a Brownian particle continuously bombarded by molecules from all sides. ${ }^{7}$ It is that coordinate that we are interested to follow in time by averaging over many molecular impacts. The averaging period must therefore be short compared to the time of coordinate variation of the Brownian particle, i.e., of the variations in local energy flows.

\subsection{Minimum information required}

The minimum data required to be collected by the sensors and reported to the central processing unit (CPU) is determined by the requirement that the CPU be capable of 
reconstructing from these data both the Graph and the State of the network. The reported parameters must include the geographical position of each sensor (see Sect. 4), the direction of the energy flow and the value of RMS current through each sensor, synchronously measured over the entire network and averaged over the chosen period.

The reported data may be restricted only to changes in the measured parameters rather than parameters themselves. This may be useful if communication bandwidth is limited.

\subsection{Communication network}

Individual sensors form a communication network that may be of different topology than the Graph of the power network itself. Specification of the nature and properties of the required sensors, as well as their positioning within the power network, constitute an essential aspect of the proposed EGMS. Topology of the sensor network is determined by the need for efficient communication with the CPU. The preferred structure of the sensor network is the so-called mesh network, where different sensors collaborate to propagate the data in the network. ${ }^{8}$

The different topologies of the communication network and the power grid itself are illustrated in Fig. 1 below.

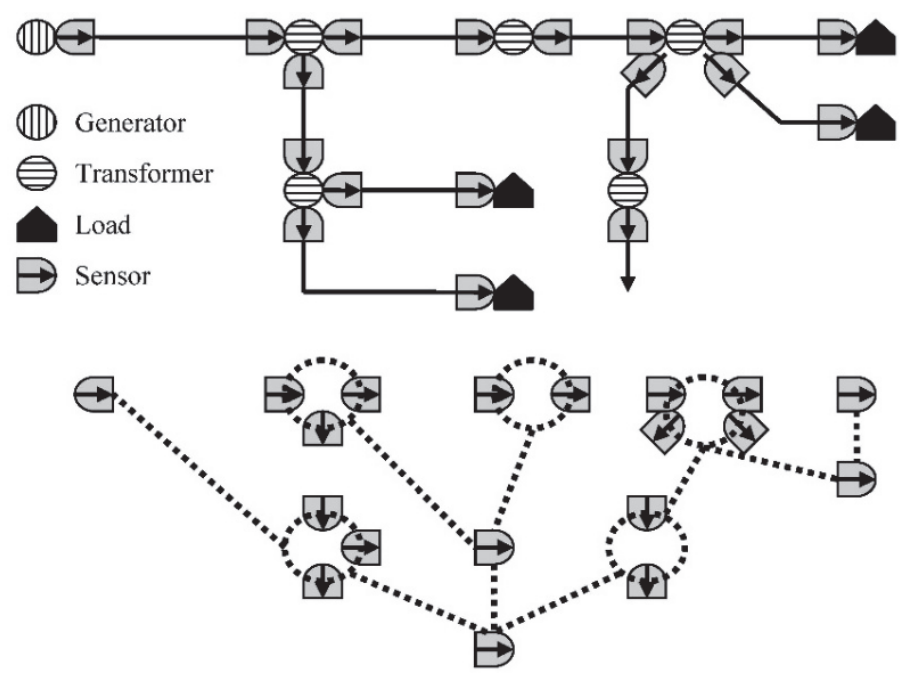

Fig. 1. Power grid (solid lines) and sensor mesh network. Established communication links are shown by the dotted lines.

\section{Kirchhoff's Law and Synchronous Averaging}

All connectivity aspects of an electric power grid are embodied in Kirchhoff's current law that must be valid at every node of the network. ${ }^{9}$

\subsection{Kirchhoff's law as a tool for network topology reconstruction}

Full description of a power grid in operation could be provided by the complete diagram 
of the energy flow. To build such a diagram one needs the knowledge of the Poynting vectors, $\mathbf{S}=\mathbf{E} \times \mathbf{H}$, in every conducting line. However, a monitoring system based on the direct measurement of the Poynting vectors would be impractical and costly. Indeed, precise determination of the electric field vector $\mathbf{E}$ requires measurement of the potential on the high-voltage wire relative to ground. One can avoid direct galvanic connection to AC wire by using cylindrical capacitive probes. However the impedance of a voltmeter arranged between these probes would have to be much larger than their impedance, i.e. at least as high as $1 \mathrm{G} \Omega$ - due to the unavoidably low capacitance of the probes. Such a voltmeter would be costly and impractical for our purpose.

Moreover, a description based entirely on the energy flow would not give full justice to connectivity of the network. Indeed, the flow of electromagnetic energy over the network does not obey a continuity equation because of dissipation.

The continuity equation that works over the power network reflects the conservation of charge and is embodied in the Kirchhoff law (for current). This suggests Kirchhoff's law as the main tool for topology reconstruction. Measurement of the current magnitude in a wire is simple and non-galvanic. In practice, it reduces to measuring the magnetic field at a distance from the wire. Consistent application of Kirchhoff's law needs only one additional ingredient, the direction. But the direction of what? The AC current has no direction and when we characterize it by a RMS value, we can "point the arrow" either way. The issue can be resolved by a simple convention to point the arrow in the direction of the energy flow. This information is encoded in the orientation of the Poynting vector.

Measurement of the orientation (and not magnitude) of the Poynting vector is a much simpler task that can be accomplished without galvanic connections. All that we need is the phase between AC current and AC voltage, without any need for the knowledge of their magnitudes. A schematic illustration how this measurement can be done with the help of a capacitive voltage divider is shown in Fig. 2. The capacitive divider comprises two insulated cylindrical probes of capacitance $C_{\mathrm{p} 1}$ and $C_{\mathrm{p} 2}$, respectively, in series with a measuring capacitor $C_{\mathrm{m}}$. The magnitude of the voltage drop on $C_{\mathrm{m}}$ is rather uncertain

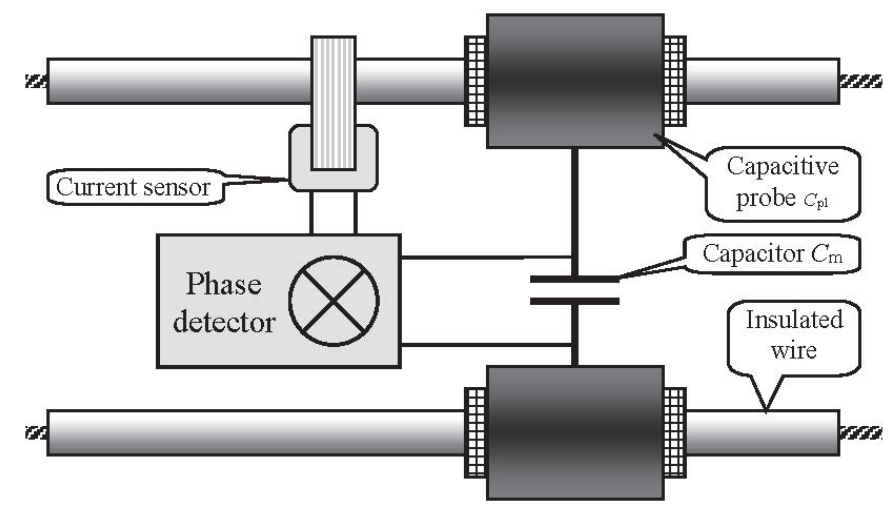

Fig. 2. Non-galvanic measurement of the direction of energy flow in AC wire. The relative phases of voltage and current are determined accurately by the capacitive voltage divider $C_{\mathrm{p} 1}-C_{\mathrm{m}}-C_{\mathrm{p} 2}$ and the current sensor, e.g. a current transformer. The capacitive divider introduces no phase-shift. 
because the capacitance of the cylindrical probes is poorly controlled but the phase of this voltage drop is monitored accurately.

Knowledge of the Poynting vector orientation in each conducting line will ensure consistent application of Kirchhoff's law.

\subsection{Kirchhoff's law for transformer nodes}

A power transformer comprises a plurality of primary and secondary coils carrying AC currents that interact magnetically. ${ }^{10}$ Each coil is an element of a circuit where Kirchhoff's law is in full obeisance. The law, however, fails to describe the relation between currents in different coils of the transformer itself, which is an extended object, not a simple point node. There are two reasons for this failure: the transformation coefficient $k_{i j}=V_{j} / V_{i} \neq 1$ between the voltages on any pair of primary and secondary coils and the power loss $\eta<1$ in the ferromagnetic core. Parameters $k_{i j}$ and $\eta$ are characteristics of any transformer, assumed known to the CPU. Knowledge of these parameters enables us to extend Kirchhoff's law to the case of a transformer by a simple generalization,

$$
\eta I_{1}=k_{12} I_{2}+k_{13} I_{3},
$$

where $I_{j}$ denote the currents flowing in and out of the transformer node, cf. Fig 3. With this generalization, Kirchhoff's law assumes a universal validity over the entire network. Each graph thus reduces to a set of homogeneous equations for the set of $\left\{I_{j}\right\}$ that any legitimate state of the graph must obey. Any tampering with the network that results in unaccounted current flow will generate a violation of this set of equations, immediately seen by the CPU with an easily pinpointed location.
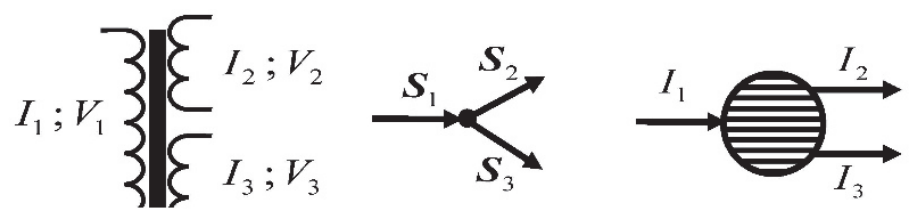

Fig. 3. Generalized Kirchhoff's law (1) for a transformer node allows one to represent the latter as a point node. In this representation, currents $I_{1}, I_{2}$, and $I_{3}$ are the RMS values of the AC currents flowing in the hot wire of the transformer coils, related by Eq. (1). By convention, we ignore the ground wire and choose the orientation of the hot-wire current $I_{j}$ as that of the corresponding Poynting vector $S_{j}$.

\subsection{Synchronous averaging}

The non-stationary situation is, of course, more relevant. As loads vary, the RMS currents themselves become functions of time, $I_{j}=I_{j}(t)$. We can average these functions over any period $\tau$ shorter than the characteristic times $T_{j}$ of their variations without losing significant information, provided this averaging does not violate Kirchhoff's law. For a single node, this will be ensured if the averaging is done over the same time interval for all connecting lines associated with it. This will remain true for the entire network, provided the averaging is done synchronously over all nodes. In the process of averaging, the "instantaneous" RMS currents $I_{j}(t)$ are replaced by the integrals, 


$$
\bar{I}_{j}(t)=\int_{t}^{t+\tau} I_{j}\left(t^{\prime}\right) d t^{\prime} .
$$

The averaging period $\tau$ is a design choice that can be optimized depending on the network conditions. The choice is a trade-off between the resolution of individual disruptive events (optimized by the shorter $\tau$ ) and the economy in the information transmitted over communication channels. In any case, we expect $\tau$ to be much longer than the AC oscillation period of $16 \mathrm{mS}$.

\subsection{Global synchronization based on GPS}

The global positioning system (GPS) is a marvel of our times. ${ }^{11,12}$ For our purposes it provides two cornerstone signals indispensible for the functioning of the EGMS, one pinpointing space, the other time. Our utilization of the spatial information (the "global positioning" proper) will be discussed in the next section (Sect. 4). Here we are interested in the time signal that will synchronize the averaging process over the entire grid.

Synchronization will delegate the task of averaging, i.e. performing the integration (2), to individual sensors, thus significantly lowering the communication budget.

The required accuracy of synchronization is not taxing at all. All we need is specification of the initial moment with a resolution better than half-period of AC oscillation $(8 \mathrm{mS})$. This task would not be easy to accomplish through the sensor network itself because of the unpredictable delays associated with its contemplated mesh nature. The GPS provides a synchronizing signal theoretically accurate to about 10 nanoseconds. However, most receivers lose accuracy in the interpretation of the signals and are only accurate to 100 nanoseconds. Either way (by 6 or 5 orders of magnitude), this precision far exceeds the required accuracy.

A convenient way of averaging the RMS signal is to integrate over exact number of periods, $N=2 \pi f \tau$ with integer $N$. This reduces the integration error and suppresses the noise associated with asynchronous interference. An appropriate circuit for this task involves a zero crossing detector, a programmable counter and an integrator.

\section{Topology and Metric}

The reported information about the distribution of the RMS currents and the directions of energy flow enables the central processing unit to reconstruct the power network both as the graph and the state of the graph. To accomplish this, the CPU will begin from any highlevel node, e.g. from the main generator, and reconstruct the graph by branching from node to node with the help of Kirchhoff's law. Note that in this way we obtain a purely topological description: we know how nodes are connected but not where they are positioned. Geographical placement of sensors will be accomplished by using the GPS.

\subsection{Positioning of nodes on a geographical map}

This represents the $2^{\text {nd }}$ coming of the GPS in our system. Our "rules of the game" (discussed further in Sect. 5), aim at making the system cheap and safe, and require self- 
organization of each sensor. The installer needs not program the sensor as to where it is installed. Each sensor learns its $x-y$ position from the GPS and uses this information for self-identification in the mesh network. The current GPS receivers provide an accuracy of order $1 \mathrm{~m}$ horizontally, which should be sufficient to distinguish sensors belonging to different nodes. Sensors belonging to the same node (on different connecting lines) will be identified as such. All sensors should be installed oriented in the same way relative to the nearest node, which will enable the identification of upstream or downstream power lines via measured orientation of the Poynting vector.

\section{2. $2 D$ and $3 D$ information}

Besides the horizontal $x$ - $y$ position, used for self-identification of sensors, the GPS provides altitude information (the $z$ coordinate). This information can be used for fast identification of falling poles. This is not a "luxury" information as it obtains free of additional charge. It will become ever more useful with increasing accuracy of the GPS.

\subsection{Theft apprehension}

The proposed monitoring system provides the technical capability for apprehension of unwelcome leaks of the power, associated, for example, with unlawful tapping of the grid by an unrecognized party. In a fully implemented version of our grid monitoring system, with sensors installed at all lines down to every user, the presence of an illegally tapped line will be seen automatically, since the illegally tapped line will have no associated sensor. The leaked power will therefore not be reported by the sensor network and the state of the power network will exhibit a violation of Kirchhoff's law. This will provide a nearly instantaneous automatic detection of the unlawful leak. In the case of an incomplete network, tampering with the power distribution at a level below the monitored level can be uncovered by comparing the total energy incoming into the last monitored node over a specified period with the sum of energies received by all legitimate customers from that node. A significant discrepancy will trigger investigation. This discovery, however, would not be instant nor automatic and this consideration may be a compelling incentive for the power grid owner to complete the sensor network down to every customer.

\section{Summary and Conclusion}

\subsection{Rules of the game}

Let us re-iterate the desiderata: cheap, safe, and sufficient. The safety and cost requirements dictate our main rules: no galvanic connection to the power lines and no on-site programming of the sensor by installers. The sufficiency requirement mandates that sensors must be able to power themselves, even if the power line where they sit is disabled. It also suggests an economy-minded attitude to the utilization of our low-capacity communication channels. This implies substantial on-site averaging. 


\subsection{What is to be measured?}

The minimum set of measured parameters includes the RMS current and the relative phase between the current and the voltage. The measured parameters are processed locally in two ways. Firstly, the direction of energy flow is determined from the phase relation. Secondly, the averages are taken over chosen time periods, their start points controlled by the GPS and their end points specified by $\tau$ as programmed.

\subsection{What is to be reported?}

Each sensor regularly reports the averaged RMS current and occasionally confirms (updates) the direction of energy flow relative to the nearest node. The update of direction is particularly important in networks that include nodes with energy storage and cogeneration functionality. Another occasionally reported update involves the geographical position and altitude as determined with the help of the GPS. The need for the latter updates is likely to be warranted only when the coordinates change (e.g. falling pole or relocated sensor). Updates of position may become subject of regular reporting for mobile nodes, such as transportable generators or loads.

\subsection{Luxury information}

This may include a variety of weather-related parameters, such as temperature, humidity, speed and direction of wind, as well as the intensity of sunlight. The co-generation and storage facilities may report the amount of available energy. The "luxury" information may include the sensor orientation relative to the gravitational and magnetic fields at the sensor location. The amount of information collected is limited by the cost of the sensor. The reporting of additional information is limited primarily by the channel capacity of the mesh communication network.

\subsection{Powering of the sensors}

In its normal operation all functions of each sensor are powered inductively from the power line itself. It is important that the sensor continues its vital functions even under emergency conditions when the power line is disabled. Each sensor must be provided with a rechargeable battery and some sensors even with environmental sources of energy, such as a solar panel or a vertical-axis windmill generator.

\subsection{Final remarks}

The proposed electrical grid monitor system is expected to provide great value to the utility that owns the electrical grid. The automated update of the network state will speed up emergency maintenance and reduce its cost. Its value to end users - i.e. us the common people - is obvious: the reduced blackout time. Our system should have a worldwide impact on reliability and availability of electric supply.

We expect that the required sensors can be manufactured in large quantities at low cost and can be installed by the regular electric utility personnel without incurring much 
additional cost. We believe that utility companies will find it useful to install these devices and may also augment their other product offerings.

\section{Acknowledgments}

We thank Dr. Satya Sharma and Dr. Peter Shkolnikov for useful discussions.

\section{References}

1. J. Northcote-Green and R. G. Wilson, Control and Automation of Electrical Power Distribution systems (CRC Press, Taylor \& Francis Group, 2007) Sect. 2.7.

2. S. Khan and J. L. Mauri, Eds., Green Networking and Communications: ICT for Sustainability (CRC Press, Taylor \& Francis Group, 2013) pp. 321-323.

3. An example of such a device, currently on the market, is THP202 Phone Alert ${ }^{\mathrm{TM}}$ by Reliance Controls (http://www.reliancecontrols.com/ProductDetail.aspx?THP202).

4. See http://en.wikipedia.org/wiki/Phasor_measurement_unit and references therein.

5. M. Ahmad, Power System State Estimation (Artech House, Boston 2013) Sect. 8.5.

6. H. Bevrani, M. Watanabe and Y. Mitani, Power System Monitoring and Control (Wiley, Hoboken, 2014) Chap. 1.

7. A. Einstein, Investigations on the Theory of Brownian Movement (Dover, New York, 1956).

8. See http://en.wikipedia.org/wiki/Mesh_networking and references therein.

9. C. R. Paul, Fundamentals of Electric Circuit Analysis (Wiley, Hoboken, 2004).

10. G. P. Shultz, Transformers and Motors (Newnes, Boston, 1989).

11. A. Noureldin, T. B. Karamat and J. Georgy, Fundamentals of Inertial Navigation, Satellitebased Positioning and their Integration (Springer, Heidelberg, 2013).

12. E. D. Kaplan and C. Hegarty, Eds., Understanding GPS: Principles and Applications, $2^{\text {nd }}$ Ed. (Artech House, Boston, 2006). 